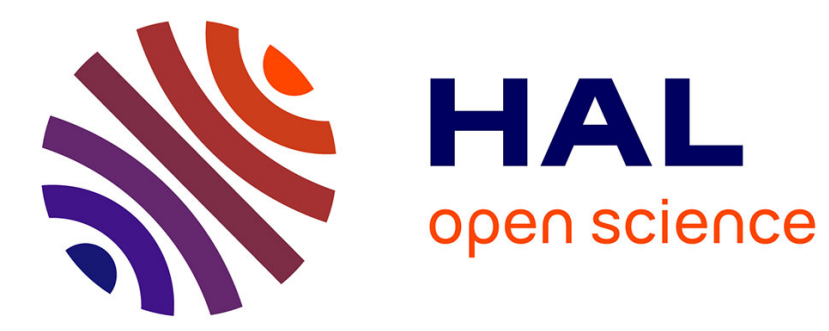

\title{
Feedback sine wave driver design for ultrasonic transducers
}

P. Schweitzer, E. Tisserand, A. Hamed, J. Andréa, F. Coutard

\section{To cite this version:}

P. Schweitzer, E. Tisserand, A. Hamed, J. Andréa, F. Coutard. Feedback sine wave driver design for ultrasonic transducers. European Physical Journal: Applied Physics, 2009, 47 (1), pp.1-7. 10.1051/epjap:2008181 . hal-00480179

\section{HAL Id: hal-00480179 https://hal.science/hal-00480179}

Submitted on 3 May 2010

HAL is a multi-disciplinary open access archive for the deposit and dissemination of scientific research documents, whether they are published or not. The documents may come from teaching and research institutions in France or abroad, or from public or private research centers.
L'archive ouverte pluridisciplinaire HAL, est destinée au dépôt et à la diffusion de documents scientifiques de niveau recherche, publiés ou non, émanant des établissements d'enseignement et de recherche français ou étrangers, des laboratoires publics ou privés. 


\title{
Feedback sine wave driver design for ultrasonic transducers
}

\author{
P. Schweitzer , E. Tisserand , A. Hamed , J. Andréa, F. Coutard \\ LIEN, University Henri Poincaré - Nancy 1, B.P. 239, \\ 54506 VANDOEUVRE-LES-NANCY, FRANCE. \\ Patrick.Schweitzer@lien.uhp-nancy.fr
}

\begin{abstract}
The optimal sinusoidal excitation of an ultrasonic transducer requests a knowledge of the frequency and the impedance of the used ceramic. These parameters, that vary during the application, depend on the characteristics of the transducer but also on the acoustic load of the propagation medium. In the search for an adaptive excitation, we propose the design of a digital generator assuring the functions of automatic tuning and impedance matching. The design uses the Butterworth-Van Dycke model of pizoelectric ceramics.

The method of determination and identification of the model parameters is presented and applied on three different transducers.

The negative feedback of the generator is carried out by the signal measured on the transducers. The dynamic voltage being very variable, the output resistor of the driver is controlled by transducer impedance.

This feedback control allows the stability of the output voltage to a constant value whatever the frequency and the medium is.

A Simulink ${ }^{\circledR}$ model of the regulation loop shows that the frequency tuning could be realized by exploiting the command signal of the driver resistance. The precision and the stability of the feedback system are tested for frequencies between 1 to $3 \mathrm{MHz}$.
\end{abstract}

Keywords : Ultrasound, feedback generator, sine wave driver.

\section{Introduction}

It is will known that ultrasonic transducers are mainly composed of piezoelectric material. To obtain the best axial resolution, in echographic imaging, the excitation is realised by a pulse discharge. In many other applications like velocimetry Doppler, non destructive control, the cutting or the thermo-welding by ultrasounds, we generally use a sinusoidal excitation in continuous or pulse mode. In this case, to obtain an effective excitation, the generator frequency must be equal to the vibration mode of the tranducer. This optimal frequency, located between the serie and parallel electric resonances of the transducer, can vary under the influence of many parameters like :

- the variations of the acoustic characteristics of the front and the back mediums [1].

- the ambient temperature.

To these variations, we can also mention the characteristics drifts related to age or fatigue of the transducer $[2,3]$.

The excitation efficiency is defined by the ratio of the acoustic power emitted by the transducer on the electric power supplied by the generator. The best ratio and the largest bandwidth are obtained when the resistance of the output driver is similar to the real part of the transducer impedance [4].

For practical applications this adaptation is difficult to realise because the transducer impedance depends on the acoustic load which can vary largely. 
In order to solve these difficulties, some generators integrate an automatic adjustment of the frequency. Others have an adjustable output resistance. In patent [5], the authors propose an automatic adjustment of the generator output resistance only for a pulsed excitation. The originality of our work lies in the automatic frequency control of the generator simultaneously realized with the control of its output resistance. A tuning ensures the automatic detection of the transducer resonance band.

The electric behaviour of a transducer with single vibration mode is represented by the Butterworth-Van Dycke (BVD) model presented in the first part. We describe the procedure to quickly determine the model parameters and the transducer impedance from the geometric and piezoelectric specifications.

After the presentation of the feedback generator principle, we have studied in the second part the influence of its output resistance on the frequential voltage dynamic applied to the transducer.

In the last part we propose an automatic control of the output driver resistance. The simulation of the closed loop is realized with Simulink ${ }^{\circledR}$ for frequencies between 1 to $3 \mathrm{MHz}$. The validation of the results are presented.

\section{Parameters and values of the BVD model}

\subsection{Parameters identification method}

The Butterworth Van Dyke electric model of a piezoelectric ceramic $[6,7,8,9]$ is represented in figure 1. We can also define frequencies ranges where the transducer can be reduced to simpler models (figure 1) [10, 11].
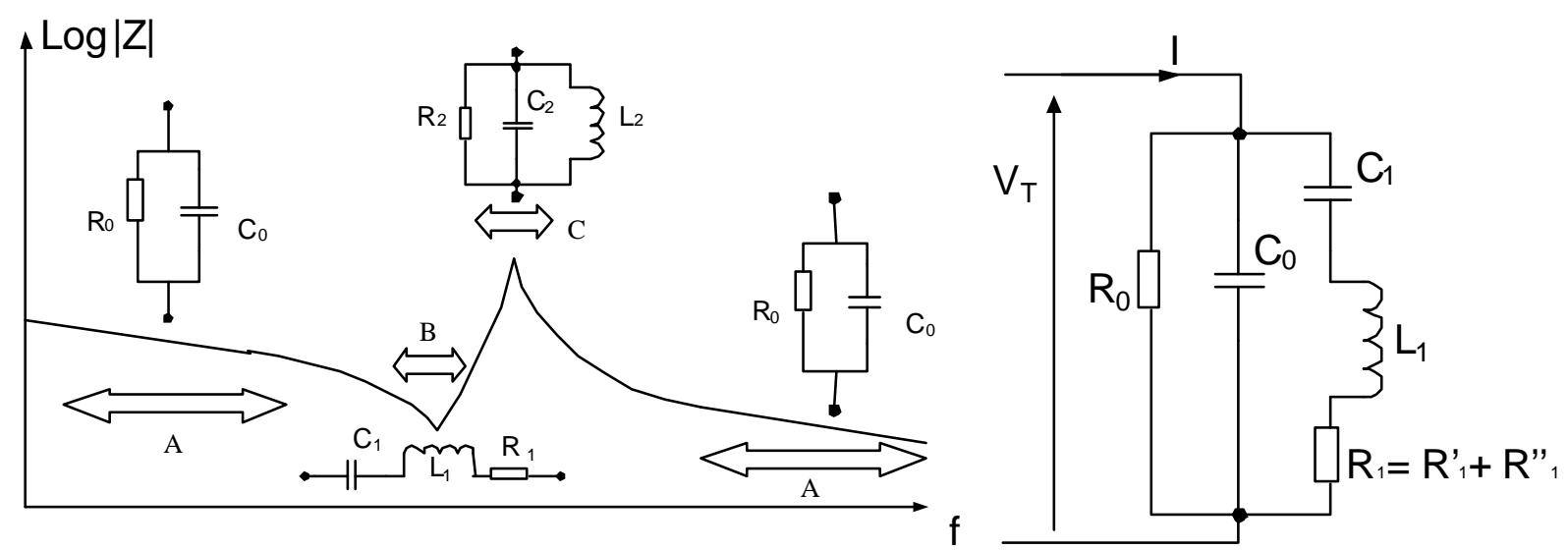

Fig 1 : Electric models of a piezoelectric transducer

The standard IEEE gives the equations permitting to determine the piezoelectric characteristics of a transducer in single vibration mode with low electric and mecanic losses $[12,13]$.

The theoretical relations to determine the model components are given in Table 1.

\begin{tabular}{|c|l|l|}
\hline Components & Theoretical relations & \multicolumn{1}{c|}{ Physical parameters } \\
\hline$C_{0}$ & $C_{0}=\frac{\varepsilon_{33}^{S} \cdot A}{d}$ & $\begin{array}{l}\varepsilon_{33}^{S}: \text { the permitivity of the piezoelectric material } \\
A: \text { the emitting surface } \\
d: \text { the thickness of the vibrating material }\end{array}$ \\
\hline$R_{0}$ & $R_{0}=\frac{1}{\omega \cdot C_{0} \cdot \tan \delta_{e}}$ & $\tan \left(\delta_{\mathrm{e}}\right):$ the dielectric loss tangent \\
\hline
\end{tabular}




\begin{tabular}{|l|l|l|}
\hline$C_{1}$ & $C_{1}=\frac{k_{e f f}^{2}}{1-k_{e f f}^{2}} \cdot C_{0}$ & $\begin{array}{l}k_{e f f}=\sqrt{\frac{8 \cdot k^{2}}{\pi^{2}}} \\
\text { resonance } \\
\mathrm{k}: \text { the global coupling factor }\end{array}$ \\
\hline$L_{1}$ & $L_{1}=\frac{1}{C_{1} \cdot \omega_{r}^{2}}$ & $\omega_{s}:$ the pulsation of serial resonance \\
\hline$R_{1}^{\prime}$ & $R_{1}^{\prime}=\frac{L \cdot \omega_{r}}{Q}$ & $\mathrm{Q}:$ the mecanic quality factor of the piezoelectric material \\
\hline$R_{1}^{\prime \prime}$ & $R_{1}^{\prime \prime}=\frac{\pi \cdot\left(Z_{B}+Z_{F}\right)}{4 \cdot k^{2} C_{0} Z_{C} \cdot \omega_{r}}$ & $\begin{array}{l}\mathrm{Z}_{\mathrm{C}}: \text { the mecanic impedance of the piezoelectric material } \\
\mathrm{Z}_{\mathrm{B}} \text { et } \mathrm{Z}_{\mathrm{F}}: \text { the mecanic impedance of the front and back propagation medium }\end{array}$ \\
\hline
\end{tabular}

Table 1 : model BVD parameters for a piezoelectric element vibrating in thickness mode.

According to the figure 1 , the BVD model represents a complex impedence equal to :

$$
Z_{\text {model_BVD }}=\frac{1}{\frac{1}{R_{0}}+j \cdot \omega \cdot C_{0}+j \cdot \omega C_{1} \cdot \frac{\left(1-\omega^{2} \cdot L_{1} \cdot C_{1}\right)-j \cdot \omega \cdot\left(R_{1}^{\prime}+R^{\prime \prime}{ }_{1}\right) \cdot C_{1}}{\left(\left(R_{1}^{\prime}+R^{\prime \prime}{ }_{1}\right) \cdot \omega \cdot C_{1}\right)^{2}+\left(1-\omega^{2} \cdot L_{1} \cdot C_{1}\right)^{2}}}
$$

The serial resonance frequencies $f_{s}$ and parallel ones $f_{p}$ associated to the impedance are written :

$$
f_{s}=\frac{1}{2 \pi \sqrt{L_{1} \cdot C_{1}}} \text { and } k_{e f f}{ }^{2}=\frac{f_{P}{ }^{2}-f_{S}{ }^{2}}{f_{P}{ }^{2}}
$$

Around the frequency $\mathrm{f}_{\mathrm{s}}$, the impedance becomes:

$$
\left|Z_{f_{S}}\right|=\frac{1}{\left(\frac{1}{R_{0}}+\frac{1}{R_{1}^{\prime}}\right)^{2}+\frac{C_{0}{ }^{2}}{L_{1} \cdot C_{1}}}
$$

For a type P1-88 ceramic at the frequency $\mathrm{f}, \mathrm{R}_{0}$ can be negligated. In this case the impedance module can be written:

$$
\left|Z_{f_{S}}\right|=\frac{\left(R_{1}^{\prime}\right)}{\left(\frac{R_{1}^{\prime} \cdot C_{0}}{\sqrt{L_{1} \cdot C_{1}}}\right)^{2}+1} \quad\left(\frac{R_{1}^{\prime} \cdot C_{0}}{\sqrt{L_{1} \cdot C_{1}}}\right)^{2} \ll 1 \text { we obtain }\left|Z_{f_{s}}\right|=R_{1}^{\prime}
$$

The resistance $\mathrm{R}_{1}{ }_{1}$ can be identified by measuring the module of the impedance at $\mathrm{f}_{\mathrm{s}}$.

The low frequency measures of $\mathrm{C}_{0}$ and $\mathrm{R}_{0}$ are carried out by an LCR meter between $1 \mathrm{kHz}$ and $10 \mathrm{kHz}$.

The value of $R_{0}$ can be estimated from the value of the impedance $Z$ at $f_{p}$ :

$$
R_{0}=\frac{1}{\frac{1}{\left|Z_{f_{P}}\right|}-\frac{C_{0} \cdot R_{1}^{\prime}}{R_{1}^{\prime 2} \cdot C_{0}+L_{1} \cdot k_{\text {eff }}{ }^{2}}}
$$

\subsection{Example}


The identification of the model components is made with a $20 * 20 \mathrm{~mm}^{2} \mathrm{P} 1-88$ transducer (T3Table 3), vibrating in thickness mode ( $2 \mathrm{MHz}$ ). The measurements of the impedance around the resonance frequencies are:

$f_{S}=1,97 \mathrm{MHz} ; f_{P}=2,234 \mathrm{MHz} ;\left|Z_{f_{S}}\right|=0,8 \Omega ;\left|Z_{f_{P}}\right|=414 \Omega ; k_{e f f}=0,47$

In low frequency the estimated value of $\mathrm{C}_{0}$ is $3.02 \mathrm{nF}$ [14].

$R_{1}^{\prime}=0,8 ; C_{1}=\frac{k_{e f f}^{2}}{1-k_{e f f}^{2}} C_{0}=0,86 n F ; L_{1}=\frac{1}{C_{1} \cdot \omega_{r}^{2}}=7,6 \mu H$ and $R_{0}=1,02 k \Omega$

In the table 2 , we can see that the identified values are near to theoretical ones.

\begin{tabular}{|c|c|c|}
\hline Parameters & $\begin{array}{c}\text { Theoretical values } \\
(\text { Table 1) }\end{array}$ & Measured values \\
\hline $\mathrm{C}_{0}$ & $2,96 \mathrm{nF}$ & $3,02 \mathrm{nF}$ \\
\hline $\mathrm{f}_{\mathrm{s}}$ & $2,02 \mathrm{MHz}$ & $1,97 \mathrm{MHz}$ \\
\hline $\mathrm{L}_{1}$ & $8,68 \mu \mathrm{H}$ & $7,6 \mu \mathrm{H}$ \\
\hline $\mathrm{C}_{1}$ & $715 \mathrm{pF}$ & $860 \mathrm{pF}$ \\
\hline $\mathrm{R}_{1}$ & $1,38 \Omega$ & $0,8 \Omega$ \\
\hline $\mathrm{R}_{0}$ & $1,85 \mathrm{k} \Omega$ & $1,02 \mathrm{k} \Omega$ \\
\hline
\end{tabular}

Table 2 Theoretical and identified values of the BVD model.

The procedure of identification, which can be generalized, is not presented for the other tested transducers.

\subsection{Transducers features}

For our experiments, we have used three different piezoelectric ceramics whose main features and complex impedance are given in Table 3 . Air is the medium of propagation .

The impedance of the transducers is obtained from the Butterworth Van Dyke's model (1). The numerical values are calculated from the theoretical equations of the components $\left(\mathrm{R}_{0}, \mathrm{C}_{0}\right.$, ...) given in table 1 .

\begin{tabular}{|c|c|c|c|}
\hline Transducer & $\begin{array}{l}\text { Geometry and } \\
\text { dimensions }\end{array}$ & Impedance of the transducers & \\
\hline $\mathrm{T} 1$ & $\begin{array}{l}\text { Thin disc } \\
\mathrm{e}=0.91 \mathrm{~mm} \\
\varnothing 20 \mathrm{~mm}\end{array}$ & $\mathrm{Z}_{1}(s)=\frac{6,18 \cdot 10^{-15} \mathrm{~s}^{2}+9 \cdot 84 \cdot 10^{-10} \mathrm{~s}+1}{1,44 \cdot 10^{-23} \mathrm{~s}^{3}+5,95 \cdot 10^{-18} \mathrm{~s}^{2}+2,89 \cdot 10^{-9} \mathrm{~s}+5,92 \cdot 10^{-4}}$ & (6) \\
\hline $\mathrm{T} 2$ & $\begin{array}{l}\text { Thin disc } \\
\mathrm{e}=1 \mathrm{~mm} \\
\varnothing 50 \mathrm{~mm}\end{array}$ & $\mathrm{Z}_{2}(s)=\frac{5,13 \cdot 10^{-15} \mathrm{~s}^{2}+8,95 \cdot 10^{-10} \mathrm{~s}+1}{8,2 \cdot 10^{-23} \mathrm{~s}^{3}+3,71 \cdot 10^{-17} \mathrm{~s}^{2}+1,98 \cdot 10^{-8} \mathrm{~s}+4,44 \cdot 10^{-3}}$ & (7) \\
\hline $\mathrm{T} 3$ & $\begin{array}{l}\text { Thin square } \\
\mathrm{e}=1 \mathrm{~mm} \\
20 \times 20 \mathrm{~mm}\end{array}$ & $\mathrm{Z}_{3}(s)=\frac{6,2 \cdot 10^{-15} \mathrm{~s}^{2}+9 \cdot 87 \cdot 10^{-17} \mathrm{~s}+1}{1,83 \cdot 10^{-23} \mathrm{~s}^{3}+6,27 \cdot 10^{-18} \mathrm{~s}^{2}+3 \cdot 68 \cdot 10^{-9} \mathrm{~s}+5,4 \cdot 10^{-4}}$ & (8) \\
\hline
\end{tabular}

Table 3. Specifications and impedance of the three transducers.

\section{Automatic tuning of the digital generator}

\subsection{The feedback generator}


For a piezoelectric system, the optimal operating frequency is located between the serial resonance frequency $\left(f_{s}\right)$ and parallel resonance frequency $\left(f_{p}\right)$ of the transducer.

Among methods of tuning the operating frequency of a piezoelectric transducer, one technique is based on a phase locked loop frequency controlled feedback generator $[15,16]$. An other method uses the admittance of the transducer as tuning parameter [17].

The solution proposed in a preceeding work $[18,19]$ is to analyse the excitation signal of the transducer [20, 21]. This one can be, under certain conditions, the image of the transducer impedance. In this study, the output resistance $R_{G}$ of the generator is set to $50 \Omega$. The structure of an automatic tuning feedback generator is presented in figure 2 .

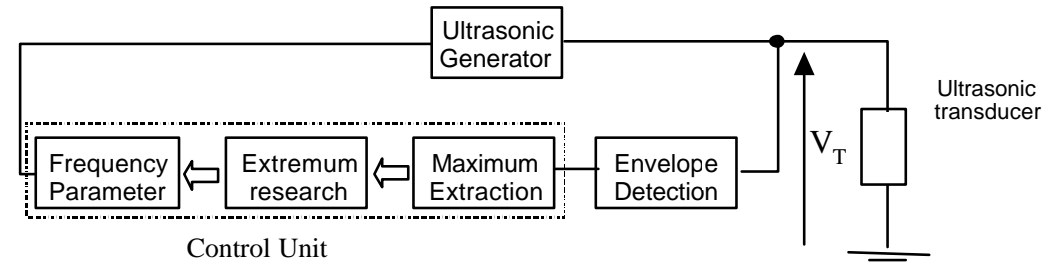

Fig 2 : Automatic tuning generator.

A control unit through a feedback detects the parallel resonance frequency for which the impedance of the transducer is maximum. The impedance module is obtained from the envelope of the excitation signal $\mathrm{V}_{\mathrm{T}}$.

A numerical algorithm uses the frequencial variations of the transducer voltage to detect the resonance band.

\subsection{Influence of the output resistance of the generator on the dynamic of $V_{T}$}

When the variations $d V_{T} / d f$ are not sufficient the detection of $f_{s}$ and $f_{p}$ is not precise. This situation can be found when the transducer impedance is too low or too high relatively with $\mathrm{R}_{\mathrm{G}}$ (figure 3).

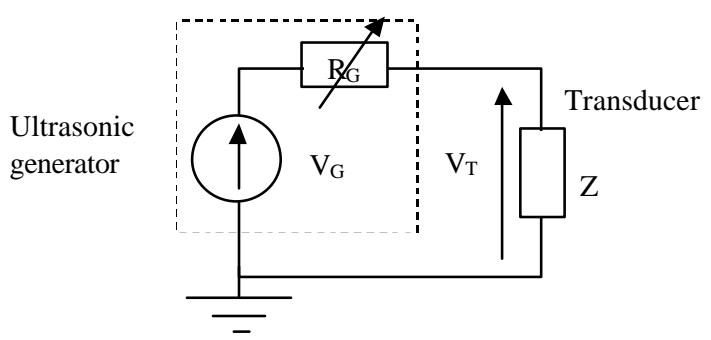

Fig 3 : Electrical circuit.

The figure 4 represents the relative voltage $V_{T}$ obtained for 4 values of $R_{G}$ for two ceramics. These theoretical figures are calculated with Matlab.

We can see that:

- A too low $R_{G}$ value makes more difficult the search for the maximal amplitude of $V_{T}(f)$.

- A too high $R_{G}$ value compresses the curve and make more difficult the search for the minimal amplitude of $\mathrm{V}_{\mathrm{T}}(\mathrm{f})$.

To resolve this problem, we propose an automatic control of the resistance $\mathrm{R}_{\mathrm{G}}$.

The second interest of this is to ensure a constant power transfer when the acoustic impedance of the medium varies. 

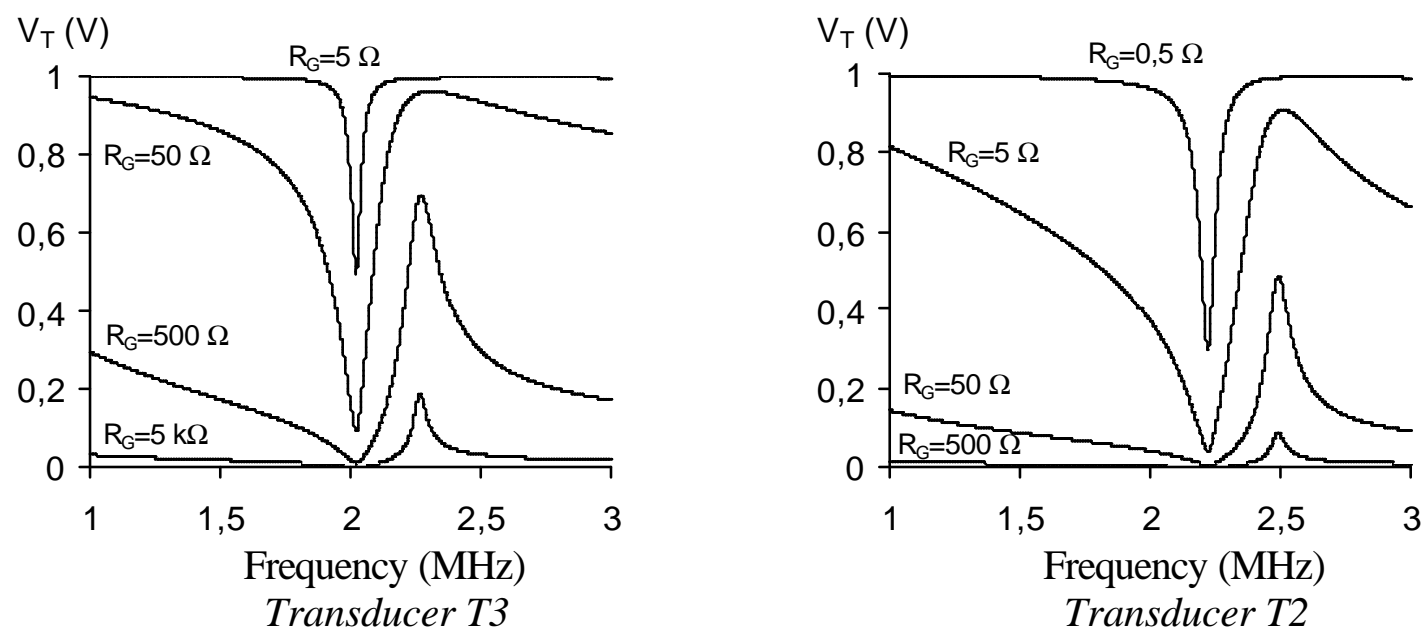

Fig $4: V_{T}(f)$ for different values of $R_{G}$

In order to verify the feasibility of the automatic control of $R_{G}$ we have simulated the regulation loop which is the subject of the following paragraph. The practical realization of the device is conditioned to the development of the generator output resistance which must be adjustable in real-time.

For that, it is possible to use a digitally ajustable resistor or a MOS active resistor.

\section{Simulation of the automatic control of the driver output resistance}

\subsection{Regulation principle}

The $\mathrm{V}_{\mathrm{T}}$ voltage is regulated at a constant value.

For $\mathrm{V}_{\mathrm{T}}=0.5 \mathrm{~V}_{\mathrm{G}}$ the best sensibility of $\mathrm{V}_{\mathrm{T}}(\mathrm{f})$ with the impedance module $|\mathrm{Z}|$ is obtained.

Indeed, by supposing that $\mathrm{Z}=\mathrm{R}$, we have:

$\frac{\mathrm{V}_{\mathrm{T}}}{\mathrm{V}_{\mathrm{G}}}=\frac{\mathrm{R}}{\mathrm{R}+\mathrm{R}_{\mathrm{G}}}$

The sensibility $\frac{\mathrm{dV}_{\mathrm{T}}}{\mathrm{dR}}=\frac{\mathrm{R}_{\mathrm{G}}}{\left(\mathrm{R}+\mathrm{R}_{\mathrm{G}}\right)^{2}}$ is maximal when $\mathrm{R}_{\mathrm{G}} \equiv \mathrm{R}$ that means $\mathrm{V}_{\mathrm{T}}=0.5 \mathrm{~V}_{\mathrm{G}}$

At the frequencies $f_{s}$ et $f_{p}$, the transducer impedance is real. Thus, at these frequencies, the condition $\mathrm{V}_{\mathrm{T}}=0.5 \mathrm{~V}_{\mathrm{G}}$ corresponds to an impedance matching which ensures a maximum transfer of electric power between the generator and the transducer.

Except these frequencies, the transducer impedance is $\mathrm{Z}=\mathrm{R}+\mathrm{jX}$

In this case, $\left|\frac{V_{T}}{V_{G}}\right|=\frac{|Z|}{\sqrt{\left(R+R_{G}\right)^{2}+X^{2}}}=\frac{|Z|}{\sqrt{|Z|^{2}+R_{G}\left(2 R+R_{G}\right)}}$

The equation $\frac{|Z|}{\sqrt{|Z|^{2}+R_{G}\left(2 R+R_{G}\right)}}=\frac{1}{2}$ has a positive solution given by:

$\mathrm{R}_{\mathrm{G}}=-\mathrm{R}+\sqrt{\mathrm{R}^{2}+3|\mathrm{Z}|^{2}}$ 
Theoretically, by regulating the voltage $V_{T}$ around the command $0.5 V_{G}$, the resistance $R_{G}$ follows the evolution of the transducer impedance module.

The scheme of the regulation is represented in figure 5.

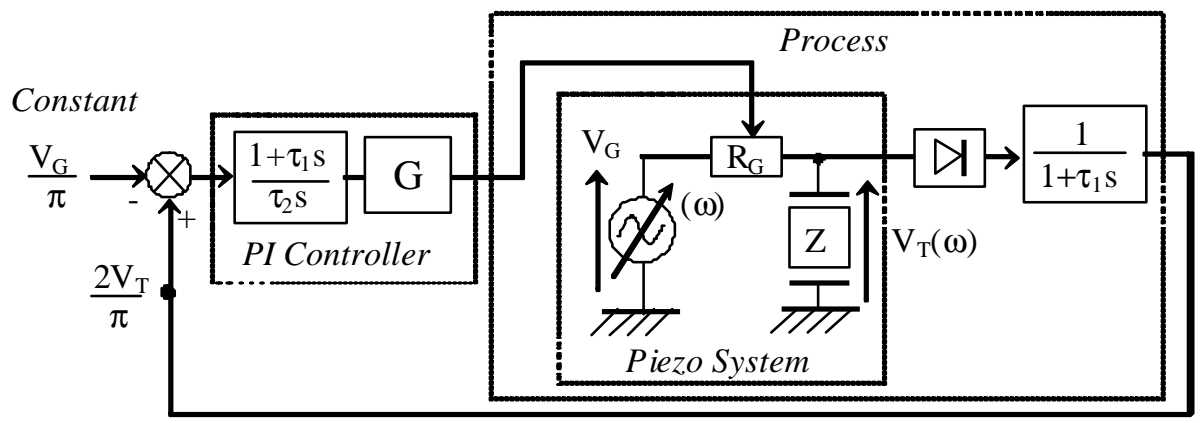

Comments

Fig 5 : Principle of the automatic resistance control.

The synthesizer generates a constant voltage $V_{G}$ with a frequency between $f_{\min }$ to $f_{\max }$.

The PI controller drives the resistance $\mathrm{R}_{\mathrm{G}}$. An increase of $\mathrm{R}_{\mathrm{G}}$ generates the decrease of $\mathrm{V}_{\mathrm{T}}$. This explains why the feedback loop is connected to the positive input and the command to the negative input of the adder.

The output $V_{T}(\omega)$ of the piezoelectric system is rectified then filtered to extract the RMS value that equals to $\frac{2 V_{T}}{\pi}$ for a rectified sinusoidal signal.

The cutoff frequency $\frac{1}{2 \pi \tau_{1}}$ of the filter is chosen equal to about $\frac{\mathrm{f}_{\min }}{100}$ to ensure a low ripple rate at the input differentiator for $\mathrm{f}_{\min }$.

The numerator $1+\tau_{1} \mathrm{~s}$ of the PI controller has a same constant of time than the low-pass filter in order to compensate his pole and to reduce the delay.

The value $\frac{G}{\tau_{2}}$ of the PI is calculated to get the best compromise between accuracy and stability of regulation.

\subsection{Simulink diagram}

We have simulated the dynamic comportment of the feedback system with Simulink ${ }^{\circledR}$. The complete simulation diagram (with $\mathrm{V}_{\mathrm{G}}=1$ ) is presented in figure 6 . 


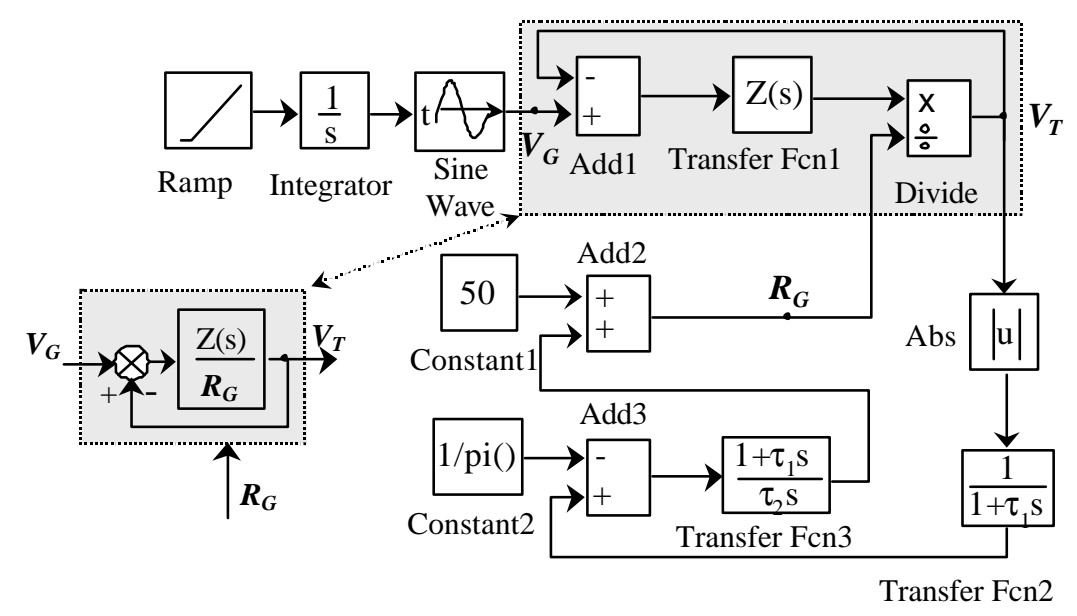

Fig 6 : Simulink diagram of the regulation.

Comparing to the original scheme, we transform the voltage divider $\frac{Z}{Z+R_{G}}$ to an implanting system with Simulink where the value $\mathrm{R}_{\mathrm{G}}$ can be isolated.

We note $\mathrm{Z}(\mathrm{s})$ the complex impedance of the transducer where s represents Laplace variable.

When writing $\frac{\mathrm{Z}(\mathrm{s})}{\mathrm{Z}(\mathrm{s})+\mathrm{R}_{\mathrm{G}}}$ in the form $\frac{\frac{\mathrm{Z}(\mathrm{s})}{\mathrm{R}_{\mathrm{G}}}}{1+\frac{\mathrm{Z}(\mathrm{s})}{\mathrm{R}_{\mathrm{G}}}}$ we obtain a secondary looped system.

The scanning frequency of the voltage $\mathrm{V}_{\mathrm{G}}$ is provided by VCO. The input signal is a ramp witch the slope adjusts the scanning rate. The initial value of resistance $R_{\mathrm{G}}$ is fixed to $50 \Omega$ in order to avoid a division by 0 at the beginning of the process.

\subsection{Experimental conditions}

We have tested the comportement of feedback system for three different transducers whose characteristics are given in table 1. These transducers have a theoretical resonance range between 2 and $2.5 \mathrm{MHz}$. We limit the frequential range of analysis between $\mathrm{f}_{\min }=1.8 \mathrm{MHz}$ and $\mathrm{f}_{\max }=2.8 \mathrm{MHz}$. Time of analysis is $10 \mathrm{~ms}$ with a100 MHz/s scanning rate.

The constant $\tau_{1}$ of the low-pass filter is fixed to $10^{-5} \mathrm{~s}$, this corresponds to a cutoff frequency of $16 \mathrm{kHz} \approx \frac{\mathrm{f}_{\min }}{112}$. The ripple frequency $\left(2 \mathrm{f}_{\min }\right)$ of the rectified signal is attenuated by about 47 $\mathrm{dB}$.

\subsection{Dynamic results.}

The dynamic behaviour of the system depends on the choice between stability and precision of $V_{T}$. This can be done by adjusting the ratio $k=\frac{G}{\tau_{2}}$. Figure 6 represents the $V_{T}$ level of the transducer $\mathrm{T} 1$ versus frequency for three values of $\mathrm{k}: \mathrm{k}_{1}=10^{6} ; \mathrm{k}_{2}=10^{7} ; \mathrm{k}_{3}=10^{8}$ and $\mathrm{k}_{4}=10^{9}$. The dynamic behaviour obtained for the two other transducers are similar. 


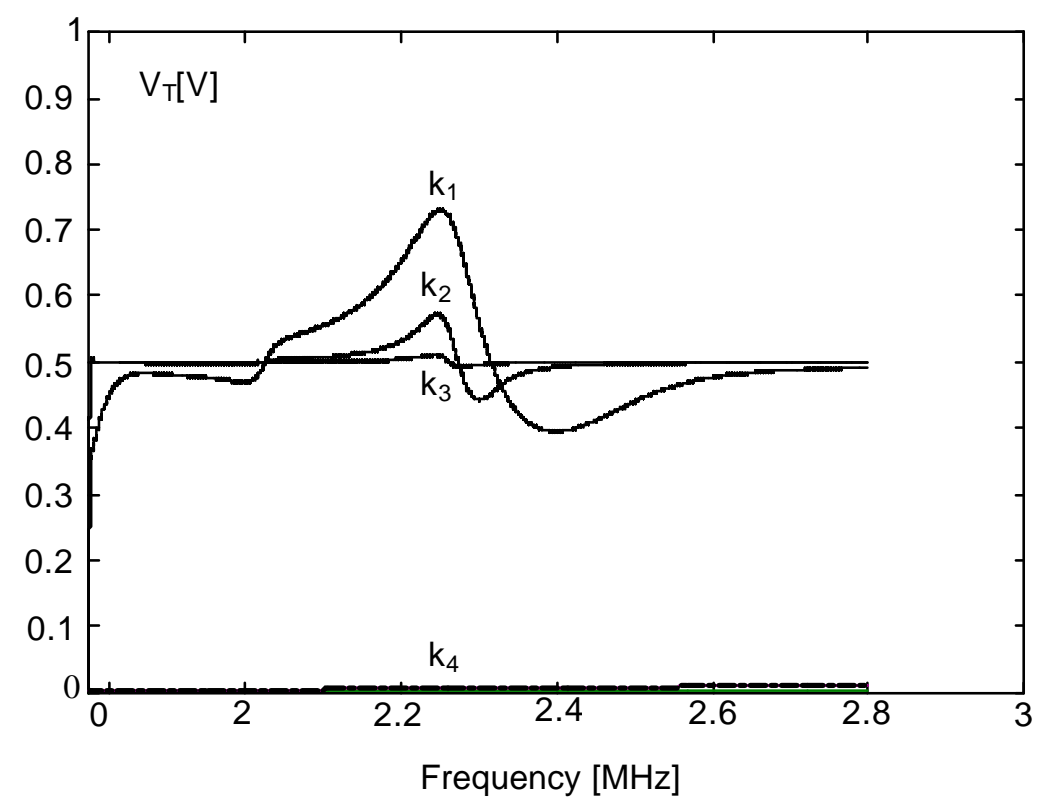

Fig 7 : Level $\mathrm{V}_{\mathrm{T}}$ of $\mathrm{T} 1$ versus frequency.

The amplitude $\mathrm{V}_{\mathrm{T}}$ is kept close to 0.5 . The differences are mainly around the antiresonance frequency $\mathrm{f}_{\mathrm{p}}(2.27 \mathrm{MHz})$. The accuracy of the feedback increases with $\mathrm{k}$. But for $\mathrm{k}$ between $10^{8}$ and $10^{9}$ the system becomes unstable (example for $\mathrm{k}_{4}$ ).

\subsection{Detection of the resonance band}

The module of the three transducer impedances, represented in figure 8 , can be compared to signals $\mathrm{R}_{\mathrm{G}}\left(\mathrm{f}\right.$ ) obtained for the three feedbacks (Figure 9) with $\tau_{1}=10^{-5}$ and $\mathrm{k}=10^{6}$.

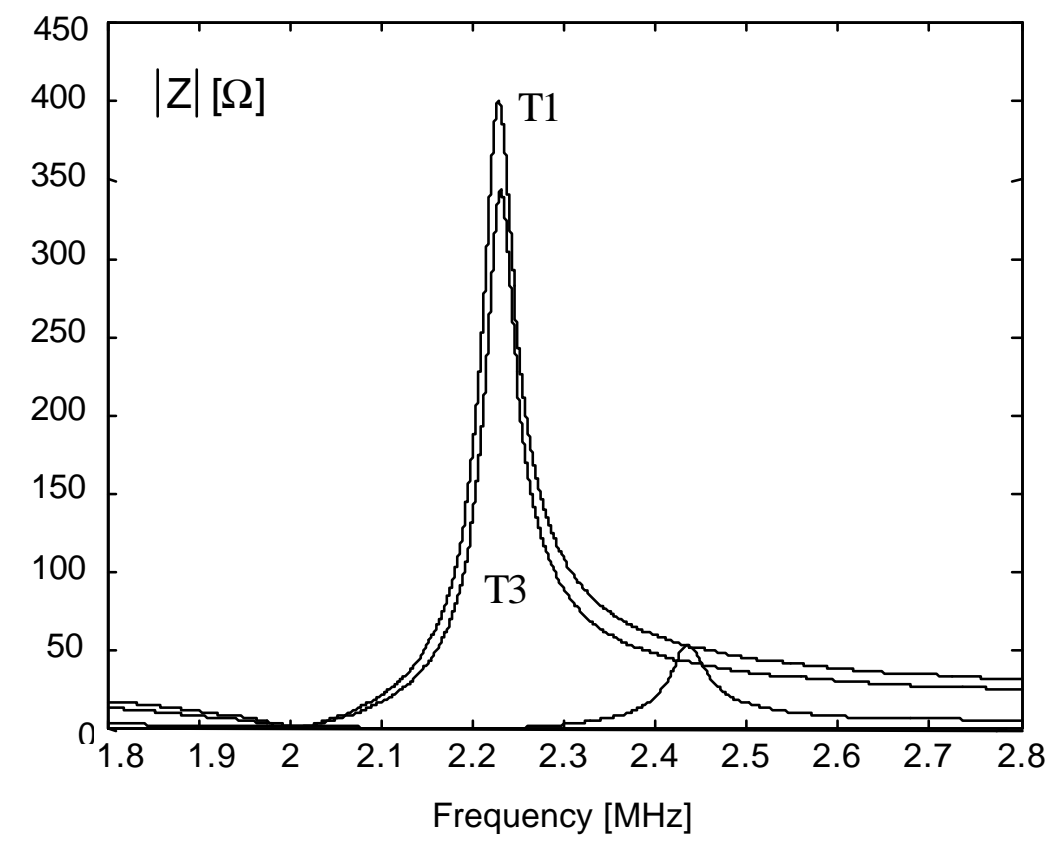

Fig 8 : Theoretical impedance modulus 


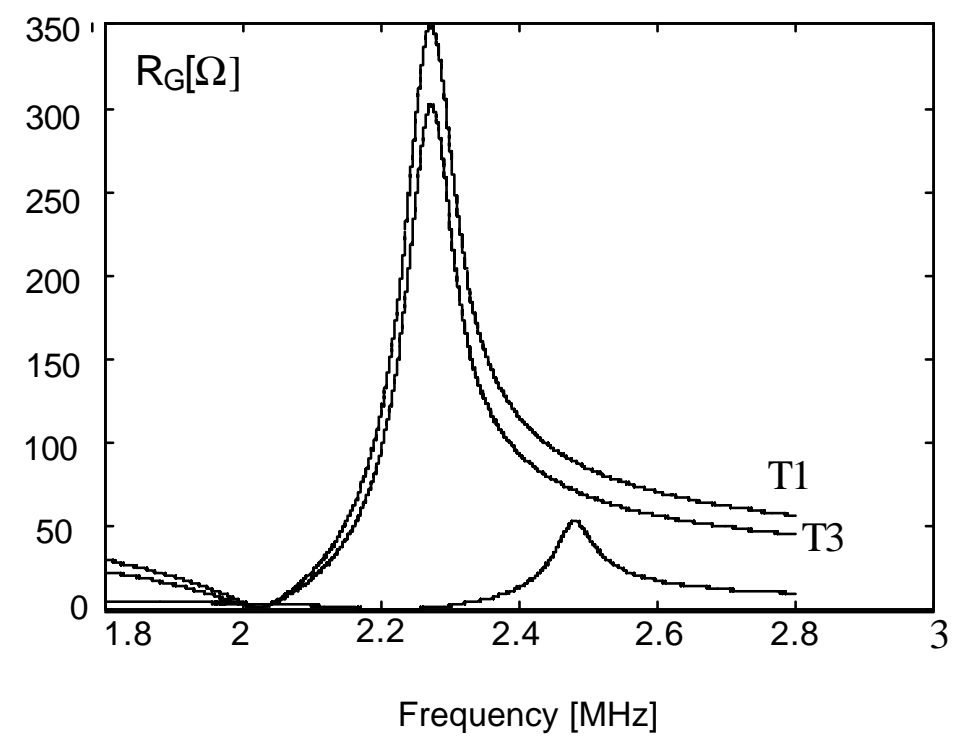

Fig $9: R_{G}(f)$ versus frequency.

We can see that the curves $|\mathrm{Z}(\mathrm{f})|$ and $\mathrm{R}_{\mathrm{G}}$ (f) are similar. In order to verify that a precise detection of the resonance is possible, we compare in table 4, frequency and impedance characteristics from the theoretical diagrams and the feedback curves.

\begin{tabular}{|c|c|c|c|c|c|}
\hline & & $\mathrm{f}_{\mathrm{s}}(\mathrm{MHz})$ & $Z_{\min }(\Omega)$ & $\mathrm{f}_{\mathrm{p}}(\mathrm{MHz})$ & $\mathrm{Z}_{\max }(\Omega)$ \\
\hline \multirow{3}{*}{$\mathrm{T} 1$} & $\begin{array}{c}\text { Theoretical } \\
\text { values }\end{array}$ & 2.011 & 1.7527 & 2.2284 & 400.1667 \\
\hline & $\begin{array}{c}\text { Simulink } \\
\text { values }\end{array}$ & 2.024482 & 1.6774 & 2.272971 & 348.5288 \\
\hline & $\begin{array}{c}\text { Relative } \\
\text { difference }(\%)\end{array}$ & -0.66 & 4.5 & -1.9 & 14.8 \\
\hline \multirow{3}{*}{$\mathrm{T} 2$} & $\begin{array}{c}\text { Theoretical } \\
\text { values }\end{array}$ & 2.1957 & 0.2313 & 2.435 & 53.3111 \\
\hline & $\begin{array}{c}\text { Simulink } \\
\text { values }\end{array}$ & 2.220882 & 0.1624 & 2.482114 & 53.2930 \\
\hline & $\begin{array}{c}\text { Relative } \\
\text { difference }(\%) \\
\end{array}$ & -1 & 42 & -1.9 & 0.03 \\
\hline \multirow{3}{*}{$\mathrm{T} 3$} & $\begin{array}{c}\text { Theoretical } \\
\text { values }\end{array}$ & 2.008 & 1.3516 & 2.2303 & 343.2421 \\
\hline & $\begin{array}{l}\text { Simulink } \\
\text { values }\end{array}$ & 2.021244 & 1.2754 & 2.273323 & 302.75 \\
\hline & $\begin{array}{c}\text { Relative } \\
\text { difference }(\%)\end{array}$ & -0.65 & 5.9 & -1.9 & 13.4 \\
\hline
\end{tabular}

Table 4 : Theoretical and measured frequencies and impedances.

These comparisons show:

- the very good agreement between the theoretical values and the simulated values.

- a high impedance dynamic (approximately $48 \mathrm{~dB}$ ) of the transducers in their range of resonance.

- These dynamic results from the low acoustic load of air

- the frequential relative variations do not exceed $1 \%$ for the resonance frequencies $\left(\mathrm{f}_{\mathrm{s}}\right)$ and $2 \%$ for frequencies antiresonance $\left(f_{p}\right)$. 
Note : for higher values of $\mathrm{k}$, the differences are lower.

These results show that the $\mathrm{V}_{\mathrm{T}}$ control allows to detect precisely the resonance of each transducer.

\section{Conclusion}

In order to excite in sinus mode a piezoelectric transducer with variable acoustic charges, we need a frequency and impedance feedback generator.

To optimize the efficiency of power transfer between the generator and transducer, we propose the design of a controlled output resistance generator. The feedback allows to maintain a constant voltage applied to transducer whatever the transducer, the used frequency and the load medium. The transducer electric model of vibration mode is a single type Butterworth Van Dyke which takes into account impedance acoustic propagation medium.

The scanning frequency permits to detect the resonance band of the transducer. The complete system is simulated in a closed loop with Simulink $®$ for three different transducers.

We see that the correction signal of $R_{G}$ is perfectly representative of the impedance module of the transducer.

The last step of this work is to realize an electronic variable resistor to test our system in a real situation.

The applications of this work concerning: the real-time control of an ultrasound system, the detection of the transducer failure, the control of the interface conditions between the transducer and the medium.

\section{References}

[1] F. Coutard, Ph.D. in Electronics, thesis, University of Nancy - France, 2007.

[2] Gerber P., Kügeler C., Schorn P. and al, Applied Physics letters. 86, 1397 (2005).

[3] Zhang Y., Lupascu DC., Balke N. and Rödel J., J. Phys. IV 128, 97 (2005).

[4] Y. Le Guerinel, R. Mallart, European Patent EP0595400, 1994.

[5] V. Loyau, G. Feuillard, J. Appl. Phys. 100, nº 034909 (2006).

[6] K.S. Van Dyke, Physical Review 25, 895 (1925).

[7] W. P. Mason, Physical Review 58, (1940).

[8] S. Sherrit, H.D. Wiederick, B. K. Mukherjee and M. Sayer, J. Phys. D: Appl. Phys. 30, 2354 (1997).

[9] A. F. Holloway, A. Nabok, M. Thompson, A. K. Ray, T.Wilkop, Sens. Acutators B 99, 360 (2004).

[10] Miodrag Prokic, Piezoelectric transducers modeling, MP Interconsulting, 2004.

[11] Dwane Rose, Load Resonant Measurement of Quartz Crystals report, Saunders \& Associates, Inc., 1989.

[12] IEEE Standard Definitions and Methods of Measurement for Piezoelectric Vibrators (177), 1966.

[13] T. L. Jordan, NASA/CR-2001-211225, ICASE Report No. 2001-28, 2001.

[14] William J. Marshall, Acoustics Research Letters Online 5, 106 (2004).

[15] J.R. Friend, D.S. Stutts, in Proceedings IEEE Ultrason. Symp. 1999, Vol. 1, p 653.

[16] A. Ramos-Fernandez, J. A. Gallego-Juarez, Ultrasonics 23, 151 (1985).

[17] W. Littmann, T. Hemsel, C. Kauczor, J. Wallaschek, and M. Sinha, in Proceedings WCU 2003, Paris, september 7-10.

[18] F. Coutard, P. Schweitzer, E. Tisserand, in Proceedings of the 19th international congress on acoustics, ICA 2007 Madrid, ISBN 84-87985-12-2. 
[19] F. Coutard, P. Schweitzer, E. Tisserand, Measurement Science and Technology 19, (2008).

[20] A. Ureche, K.D. Leukanech, J. Acoust. Soc. Amer. 2, 686 (1996).

[21] A. Arnau, T. Sogorb, Y. Jimenez, IEEE Trans. Ultrason., Ferroelect., Freq. Contr. 48 n $^{\circ}$ 2, 617 (2001). 